\title{
L.I.M.E. A recommendation model for informal and formal learning, engaged
}

\author{
Daniel Burgos \\ UNIR Research, International University of La Rioja. Spain
}

\begin{abstract}
In current eLearning models and implementations (e.g. Learning Management Systems-LMS) there is a lack of engagement between formal and informal activities. Furthermore, the online methodology focuses on a standard set of units of learning and learning objects, along with pre-defined tests, and collateral resources like, i.e. discussion fora and message wall. They miss the huge potential of learning via the interlacement of social networks, LMS and external sources. Thanks to user behaviour, user interaction, and personalised counselling by a tutor, learning performance can be improved. We design and develop an adaptation eLearning model for restricted social networks, which supports this approach. In addition, we build an eLearning module that implements this conceptual model in a real application case, and present the preliminary analysis and positive results.
\end{abstract}

Keywords - Technology-enhanced Learning, eLearning, Personalization, Social Network, Conceptual Educational Model

\section{INTRODUCTION}

Social networks focused on a specific topic or community are a powerful and precise means for user communication and interconnectivity, no matter the role they stand for. These can be learners, teachers, employees, staff, academic managers, or financial directors, who show a very determined attitude, depending on their context and their objectives. Every user can question, answer, start an activity, follow another, comment on someone else's job, score a job made by others, search onto Internet, follow a scheduled test, participate in a video-conference with a teacher, and so on. And, in all these activities, any user can be pro-active, reactive, passive, consumer, producer, dealer, and yet to show some additional facets.

To this extent, we design and develop a conceptual model, L.I.M.E. as for Learning, Interaction, Mentoring, Evaluation. These four vectors are measured and analysed as the pillars for the learning scenario, and they are depicted in various inputs which feed the model. Furthermore, we implement this model in a learning ecosystem, restricted by user access and topic. This implementation of the personalised learning model, which deals with every single input and feature aforementioned, provides the user with adaptive tutoring, thanks to a rule system. In this ecosystem, the users interact one with each other, and with the system, and they get personalised counselling.
Before and after the design and implementation of the L.I.M.E. model as a case study, we have carried out a hybrid approach mainly with qualitative studies, supported by some additional quantitative studies, with various groups of experts and end-users. Hence, we have designed and executed a Delphi study to retrieve and categorize the user requirements, as well as a number of semi-structured interviews. Furthermore, we have organized two focus groups with different experts, and one quantitative questionnaire with the students involved in the application case. In addition, we have elaborated a comprehensive state-of-the-art which combines cross-engaged topics for eLearning processes like, i.e. Education, Communication and Technology.

It is proven that the learning itinerary provided by the L.I.M.E. model is efficient and effective, and therefore, it increases the user performance. To show this approach, we have designed and implemented a learning scenario in a real class, which we have split in two groups (experimental and control) of 24 students, each. We have selected and analysed a subject of an official university online programme, during 4 weeks. This scenario engaged formal and informal activities with a comprehensive approach. The implementation shows successful results which prove the validity of the model. In addition, we have got useful recommendations and promising conclusions for further versions of the model, out of the rounds of expert and end-user consultations.

The combination of 48 learners, along 4 weeks and related milestones, the measurement of 30 inputs focused on informal and formal settings and distributed along the four main vectors, has resulted in a large dataset with sufficient information to retrieve meaningful and significant interpretation. The main outcome highlights that there is a clear and positive influence in the user performance, when the L.I.M.E. model is implemented. Furthermore, L.I.M.E. shows to be effective and efficient. This conclusion is supported by a $10,53 \%$ overall average difference between the experimental group and the control group $(66,72 \%-56,19 \%)$, with a peak difference between corners of $37,37 \%(81,41 \%-44,04 \%)$. These overall results, along with the partial ones which are presented along this research, support seamlessly the online personalised learning model for thematic, restricted social networks, L.I.M.E. 


\section{CURRENT DEVELOPMENTS AND ADDED VALUE}

In most of the e-learning environment designs, interaction and behavioral strategies have generally been neglected and therefore satisfactory uses of these strategies have rarely been realized, so that informal and formal settings are not engaged in a combined approach. Most learners are not even aware of what they have been studying (Kurt, 2007). Even when students monitor their learning, there is a broad theoretical notion that students experience illusions of competence [1], which leads to inaccurate judgment of their learning progresses and outcomes [2, 3]. For these reasons, learners need to be guided towards reflecting on their learning and improving their cognitive models of expertise. For instance, with the use of meta-cognitive expertise, which becomes crucial [4] in fostering individual's awareness of different cognitive, social, emotional, and meta-cognitive capabilities that are needed, knowledge of when and why they are useful, as well as development of regulatory skills, such as planning, monitoring, and reflecting.

Another approach makes use of recommendation settings. A recommender system is a tool that helps users to identify interesting items from a large pool of objects. It has been widely used in many commercial sites for recommending books, movies, CDs, and news articles (e.g., [5, 6]). Meanwhile, the success of these implementations has been inspiring for e-learning researchers. Multiple efforts have been made to design educational recommendation systems to recommend quality learning resources to learners to help reduce cognitive load and improve learning efficiency [7-10]. However, recommendations alone do not ensure learning performance, and how learners respond to the recommended resources defines the critical part of successful learning. Furthermore, Recommendation Systems emerge as a solution to find the right, personalized information in electronic commerce, knowledge management systems, learning management systems, social networks (open and restricted), and other fields and markets. To this extent, there are various inputs which can be used as information sources like i.e. user similarities with other users, user profile, user preferences, user behavior, user interaction, user ratings, and many other user tracking inputs $[11,12]$. All these inputs provide the system and the teacher with valuable data to recommend a personalized learning itinerary and feedback.

Other sources of information are i.e. user interests, goals, and objectives, all of them more useful for educational applications. However, current educational applications lack of enough amounts of data to establish user similarities in a precise way. In this case, recommendations are based on information stored in a user model which is extended explicitly or implicitly. There are also hybrid approaches which ask some minimum information to the user and the rest is obtained in an implicit way, but none of them engage formal and informal learning in a combined model, since expressing user preferences, behavior, interaction, goals and interest with rules can be difficult, in general. Large amounts of data are required to narrow down the recommendation, although this solution comes along with an additional problem: the size and complexity of the rule-set can be unaffordable, and inconsistencies may appear.

In this paper, we design an eLearning model for personalized learning, with special focus on the combination of formal and informal settings in a combined paradigm. In doing so, we cope with the artificial difference between Learning Management Systems and specific, restricted social networks which complement the user formal activity with informal interaction.

\section{DESCRIPTION OF THE LIME MODEL}

The L.I.M.E. model is based on three vectors:

- What every learner does based on his/her own contribution ( $\mathbf{L}=$ Learning)

- What the learner does to support interaction based and the relation with others, in addition to group interaction (I=Interaction)

- $\quad$ and what the teachers/experts value (M=Mentoring)

- In addition, there is a forth, transversal vector, being applied to the three previous vectors, focused on evaluation ( $\mathbf{E}=\mathbf{E v a l u a t i o n})$

- being the final acronym L.I.M.E., as of Learning, Interaction, Mentoring, and Evaluation

In doing so, we take into consideration every single main role in the model (i.e. the learner -individual, group-, the teacher, the expert, and the designer), as well as the main factors for a fine adaptation, such as, i.e. the learner's performance, the group's performance, trust, and reputation. In addition, this model is based on the knowledge structure depicted in the beginning of this section that consists of LE (Learning Environment), LO (Learning Object), UK (Unit of Knowledge), and PLN (Personal Learning Network).

In order to define the best setting, the model designer (e.g. teacher) must design a strategy and (s)he should follow a stepto-step process to select a number of key elements of the model:

Setting: Balance between formal and informal settings: the system collects specific inputs from both settings, keeping an overall balance of $100 \%$. For instance, if the designer requires just a formal setting, the balance should be Informal:100\% - Formal: 0\%

- Category: Balance between Learning, Interaction, and Mentoring categories: In the L.I.M.E. model, every category is assigned with a specific weight, keeping an overall balance of $100 \%$ Watch that Evaluation is a cross-category. For instance, if individual and group actions matter alike, and there is no mentoring, the balance should be Learning: $50 \%$ Interaction: 50\% - Mentoring: 0\%

- Input: List of specific inputs for each category and assigned weight: every input should reflect a number of diverse types of potential interaction and-or actions from the user to the community, and vice versa.

As an example, we provide a form with the following parameters:

Informal: 40\%. Rationale: informal activities matter, however they are not enough to pass 
- Formal: 60\%. Rationale: formal activities (e.g. exam) are key to pass, however, informal activities are required to achieve an optimum score

- Learning: 40\%. Rationale: individual activities are key in this setting, however they are not enough to pass

- Interaction: $30 \%$. Rationale: interaction itself is not enough to pass, although combined with Mentoring and-or Learning, become the key for success

- Mentoring: 30\%. Rationale: just mentoring is not sufficient, however mentoring inputs provide they key to pass, along with learning or interaction inputs

Specific inputs: as listed, looking for a fine distribution between individual and groups actions; pro-active and reactive actions; personal-group-mentoring inputs; formal and informal contribution. This list is not exhaustive, but tentative, and provides a set of inputs based on the analysis of user requirements. These inputs have an assigned weight. These weights are shown as an example, and they should be designed and adapted by the designer based on specific requirements and objectives. For clarity's shake, in the following tables, we depict the weight in three columns, showing the Absolute value (Abs), the Relative value (Rel 100), taken as $100 \%$ for every Category, and the final Relative value (Rel 40), related to the specific value of the Setting, Category, and Input, taken as $100 \%$ for the three Categories (L.I.M.E.). This last column shows the actual values for the final calculation. The Evaluation (E) vector is included in every other vector, and relies on their needs. For instance, in the following table, Evaluation is included in L=Learning (External examination, External continuous evaluation, External essay, External degree thesis), and $\mathrm{M}=$ Mentoring (Quantitative assessment, Qualitative assessment). However, these inputs (sub-vectors) might be different, based on the specific model applied to a scenario

\section{DEVELOPMENT OF THE ELEARNING PLATFORM}

In order to test and evaluate the L.I.M.E. model, we have developed a software application (i-LIME) to be implemented in a learning scenario, as it will be described afterwards. This application is supported by the Learning Management System of the International University or La Rioja (www.unir.net, http://research.unir.net) and it does not intend to be exploitable, but a prototype, since the final objective of this research is not oriented to programming but to the correct application of the model itself.

i-LIME is a learning environment (LE), built to apply the LIME model, based on Learning, Interaction, Mentoring, and Evaluation. It can be played stand-alone or integrated with another existing LE (e.g. Moodle), via web services. This platform is envisaged as a new cognitive learning concept to create, share and reuse scalable didactic content (Learning Objects, Units of Knowledge), to adapt the content to learners' individual needs, and to share with others (Personal Learning Network), according to the LIME model. In this context, the user becomes consumer and producer at the same time, the minimum unit of learning is based on a variety of resources. User education is also boosted, allowing a) more active participation in the learning process, b) objective teaching skills assessment, and c) encouraging collaboration with other teachers and tutors and trainees with different expertise.

i-LIME combines the use of didactic contents, and knowledge and learning resources, for online teaching (OT). We develop i-LIME as a technology-enhanced learning (TEL) platform which applies the LIME model, and which will facilitate a more interactive, personalized learning process. iLIME enhances the user's experience (e.g. teachers and tutors) using a five-pillared architecture [13]: (1) an authoring tool of Learning Objects and Units of Knowledge ; (2) a content management system that incorporates a modular and scalable system to capture, catalogue, search and retrieve multimedia content; (3) an adaptation management system which retrieves information and inputs from the users and the system and provides specific, personalized recommendations for the learning itinerary, based on the LIME model; (4) an evaluation module, which in turn is used as an additional input to the LIME model and the recommender layer [14]; and (5) a social thematic network (restricted to registered users in the same field) for collaborative learning between users, which provides input date on behaviour and interaction to the LIME model and the recommender layer. To this extent, we have installed an instance of i-LIME, fully operational with regards to user inputs, data collection and analysis, and adaptation management system (3, following the afore notation), along with generic functions for end-users.

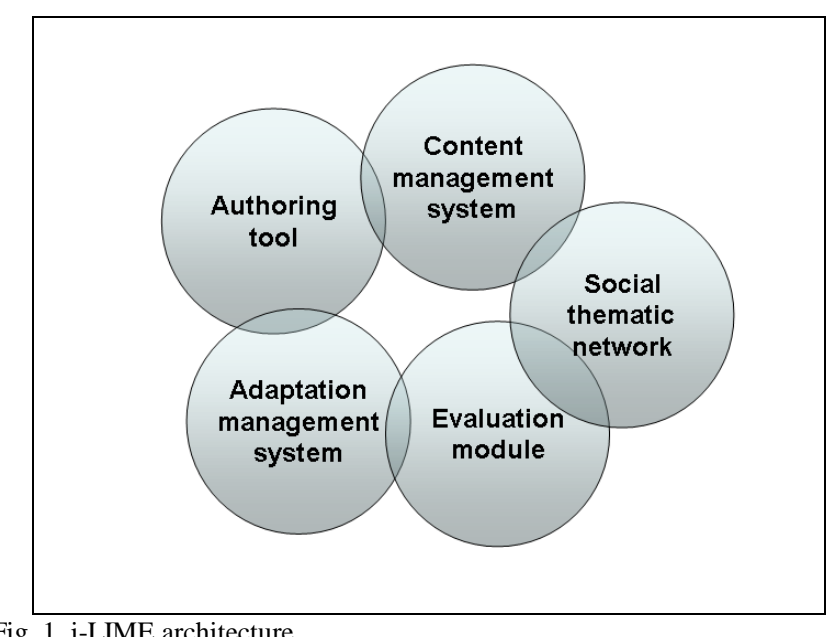

The first pillar, the authoring tool for learning objects and units of knowledge (ATH), allows the building of scalable didactic content from individual users' knowledge by means of training resources (e.g. video footage) to enhance didactic information. The content management system (CMS) works with units of information, in the form of text, video, and audio files, or any other format required to provide useful learning objects.

Users' knowledge management is achieved within the second pillar. The adaptation management system (AMS) provides adaptive learning to users based on their progress (formal learning), behavior (informal learning), and other inputs, within their continuous formative path using the environment. Recommendations are given to users regarding 
(1) the most suitable contents, (2) colleagues working in the same field, (3) interactions to perform, and (4) given their personal interests and progress in i-LIME, amongst other inputs. To this extent, the adaptation management system makes use of the Recommendation Layer (Meta-Mender), which uses meta-rules in order to provide a new abstraction level suitable for increasing personalization and adaptation.

An important matter when developing a new training process is to develop new objective evaluation systems based on reliable and measurable data, which allow for automatic and immediate feedback and which are always available for trainees. Thus, objective evaluation is a key issue in the iLIME environment. Thanks to the evaluation module (EVAL), trainees are able to test their knowledge via closed exercises, which are immediately analyzed by the environment, and used to provide input to the Recommendation Layer (Meta-Mender). Formative feedback is provided to trainees by means of corrections and future didactic content recommendations.

The final pillar in i-LIME is the social network (SN), a thematic network restricted to registered members, which allows for the creation of collaborative networks of students and professionals and provides a space where users can debate and work together. In doing so, informal learning is encouraged continuously, and the social network provides feedback to the Recommendation Layer (Meta-Mender), which will return a more accurate, personalized tutoring.

\section{V.APPLICATION SCENARIO: INFORMAL AND FORMAL LEARNING,} ENGAGED

The scenario consists of a Learning Environment (LE) adapted to a specific subject that compiles learning resources, tasks, and interactive activities, for future online teachers and tutors. These teachers and tutors have to get up-to-speed with techniques, processes, and strategies to foster, encourage, and facilitate actual learning and a clear methodology between the students. We integrate the i-LIME system in the Learning Environment of UNIR (UNIR LE), and hence the scenario is supported by two components: 1) the Virtual Campus at UNIR (UNIR LE), in which all the degrees lean on, and it is very much focused on daily administrative issues and scheduled events and activities (the formal component); and 2) the iLIME component (the informal component, namely the Adaptation Management System-AMS) (Figure 2). This technical setting supports the open interaction between peers and between other target groups (i.e. learners, teachers, tutors, admin staff, et cetera). The overall system does require the following minimum software on the client side: Windows XP/7 or Mac OS X 10.x, Firefox 13.x or Explorer 8.x (both with Javascript habilitated). On the server side: the UNIR LE, Drools Engine, Microsoft Excel, PHP 5.x, Apache 2.x.

Our learning scenario (e.g. case study) was deployed from July $2^{\text {nd }}$, to July, the $29^{\text {th }}, 2012$. To this regard, we used a graduate course on "Design and management of research projects", in the Master of Science in eLearning and Social Networks, an online, official master degree at the International University of La Rioja (UNIR). This course took place between July, 2nd and July, the 26th, 2012, with 49 enrolled students. All the students but 1 took part in the experiment. Therefore, we count 48 graduate students, between 35 and 45 years old, from 2 countries (Spain -45 students-, Colombia -3 students-) and 2 continents (Europe, South America), with a gender distribution of 28 females and 20 males.

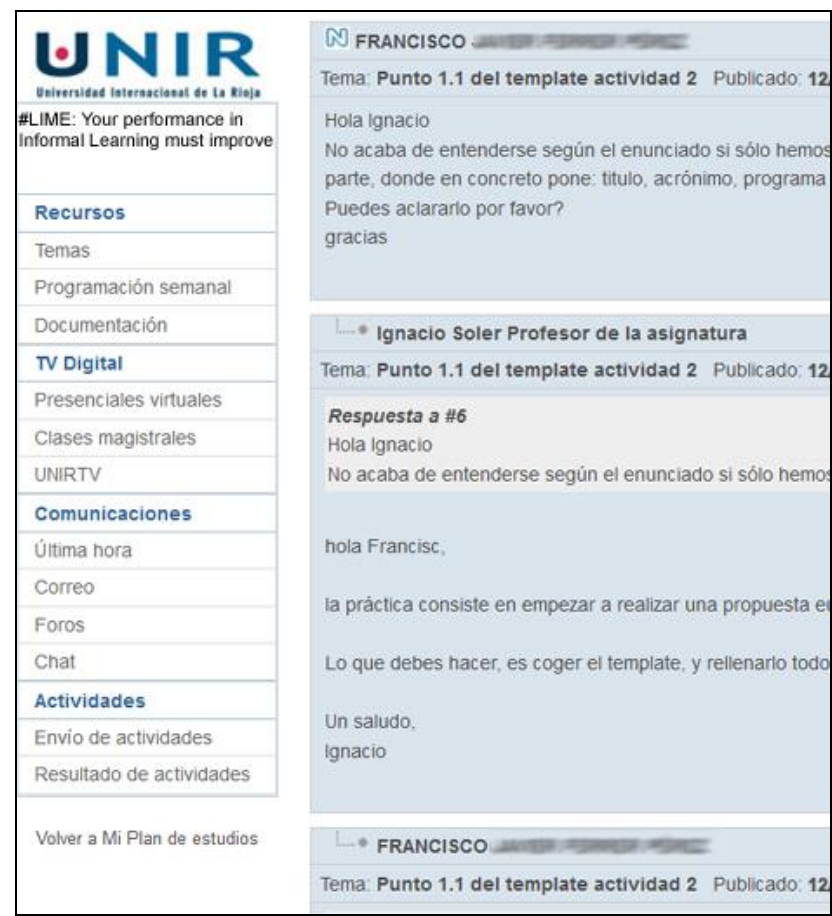

Fig. 2. Screenshot of the UNIR LE engaged with the i-LIME component

The support group consists of a teacher, an online tutor, an academic coordinator, and a master director. In addition, other cross-support departments might provide some assistant (i.e. administrative, legal, counseling, research, library, stages, et cetera). The environment is executed by every user only if the (s)he agrees with the terms described in a formal document, so that the recording of the their private date and tracking are explicitly authorized.

We have split the base group in two, equally distributed (24 members for each group). Group A (experimental) is engaged with the LIME model and receives personalized recommendation based on a number of inputs, including traditional (e.g. teacher, tutor, admin staff). Group B (control) follows the course, without the LIME model, and receives traditional support only. To make a balanced distribution of Groups A and B, on order to achieve a similar starting point, we take the previous results and evaluation. This master degree deploys the subjects in the academic program sequentially, and 9 subjects have already carried out. Therefore, there is a statistical information, quite valuable to evenly distribute members between groups (control and experimental). The final distribution is shown in Table 3 and Figure 3:

This distribution works with the individual average score after 9 subjects, out of 10-point maximum. It splits the final score of every group member in Formal (e.g. presence examination) and Informal (i.e. auto-tests, participation in 
online lectures, et cetera). Formal takes $60 \%$ of the final score; Formal provides $40 \%$ to the final score, based on a total of $100 \%$. According to

TABLE 3: SAMPLE DISTRIBUTION BASED ON PREVIOUS ACADEMIC RECORDS

\begin{tabular}{|c|c|l|c|c|c|}
\hline Group & Sample & Distribution & Formal & Informal & Total \\
\hline A & 24 & Average Group A: Experimental & 4,13 & 3,73 & 7,86 \\
\hline A & 24 & Maximum Group A & 5,80 & 4,00 & 9,80 \\
\hline A & 24 & Minimum Group A & 3,00 & 2,40 & 5,40 \\
\hline A & 24 & Standard Deviation Group A & 0,81 & 0,53 & 1,19 \\
\hline \hline B & 24 & Average Group B: Control & 4,05 & 3,69 & 7,63 \\
\hline B & 24 & Maximum Group A & 5,60 & 4,00 & 9,60 \\
\hline B & 24 & Minimum Group A & 1,70 & 2,40 & 1,70 \\
\hline B & 24 & Standard Deviation Group A & 0,88 & 0,58 & 1,67 \\
\hline \hline Total & 48 & Average Total Sample & 4,09 & 3,71 & 7,75 \\
\hline Total & 48 & Maximum Total Sample & 5,80 & 4,00 & 9,80 \\
\hline Total & 48 & Minimum Total Sample & 1,70 & 2,40 & 1,70 \\
\hline Total & 48 & Standard Deviation Total Sample & 0,83 & 0,55 & 1,44 \\
\hline
\end{tabular}

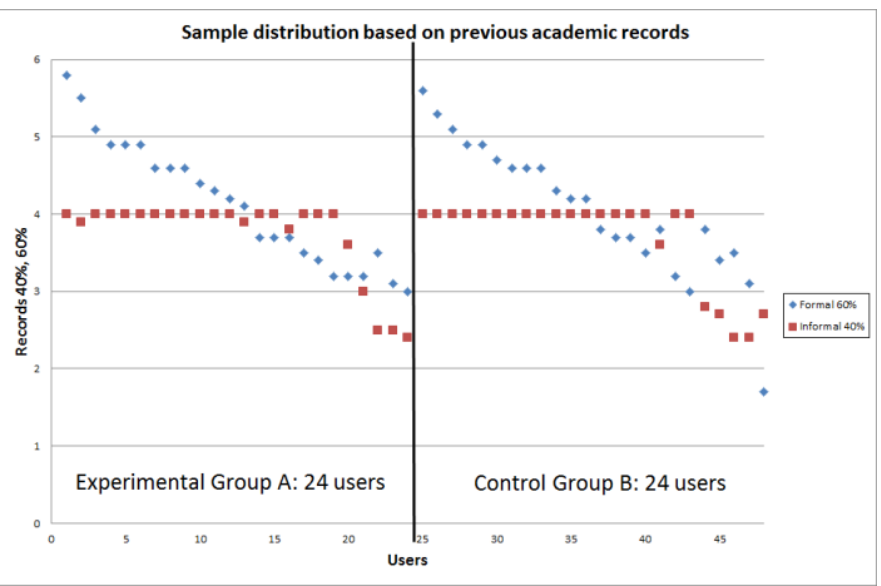

Fig. 3. Sample distribution based on previous academic records

This distribution works with the individual average score after 9 subjects, out of 10-point maximum. It splits the final score of every group member in Formal (e.g. presence examination) and Informal (i.e. auto-tests, participation in online lectures, et cetera). Formal takes $60 \%$ of the final score; Formal provides $40 \%$ to the final score, based on a total of $100 \%$. According to the data provided in Table 3 , there is a balance between Groups A and B that shows similar scores in every category, although the standard deviation is slightly different (1,19 in Group A versus 1,67 in Group B). This difference comes from a single member in Group B, who scores the minimum $(1,7)$, while the previous one scores a total of 5,5. We can conclude that the starting point for both groups is quite similar, so that the experiment starts in the same context.

\section{LIME MODEL APPLIED TO THE LEARNING SCENARIO}

With regards to the LIME model, we follow the pattern Informal50-L40-I40-M20, which the following basic rationale: "Informal and formal settings matter alike. Inputs from the user and the group make $80 \%$ of the total, being Mentoring actions taken as support and collateral ones. The Learning Environment (LE) is taken as the learning and communication platform, as well as the summative and formative resource for assessment". This model allows for an optimum adaptation to the features of the Learning Environment at UNIR, since combines formal and informal contexts, and supports self- learning and learning from others, including mentors (i.e. teacher and tutor). In addition, this pattern encourages the use of Units of Knowledge (UK, made of Learning Objects combined with complementary information), and Personal Learning Network (PLN, made of LO and UK, along with all the interaction elicited from other users).

Based on this model, the level of integration with UNIR's Learning Environment, the learner sample, the subject, and the overall objective, we have defined a set of Inputs, which will be used as a base to write the appropriate adaptation rules that will feed the LIME model (Figure 4Fig):

\begin{tabular}{|c|c|c|c|c|c|c|}
\hline \multirow[t]{2}{*}{ ID } & \multirow{2}{*}{\multicolumn{2}{|c|}{ Category Setting }} & \multirow[t]{2}{*}{ Input } & \multicolumn{3}{|c|}{ Weight } \\
\hline & & & & Abs & Rel 100 & Rel 40 \\
\hline & Le & & Input Learning Subtotal & $100,00 \%$ & $100,00 \%$ & $40,00 \%$ \\
\hline & & & Input Learning Informal & $50,00 \%$ & $50,00 \%$ & $20,00 \%$ \\
\hline & & & Input Learning Formal & $50,00 \%$ & $50,00 \%$ & $20,00 \%$ \\
\hline & & & Subtotal Learning & $220,00 \%$ & $100,00 \%$ & $40,00 \%$ \\
\hline & & & Subtotal Learning Informal & $140,00 \%$ & $50,00 \%$ & $20,00 \%$ \\
\hline & & & Subtotal Learning Formal & $80,00 \%$ & $50,00 \%$ & $20,00 \%$ \\
\hline$\overline{\mathrm{L} 1}$ & Learning & Informal & Post a message & $10,00 \%$ & $3,57 \%$ & $1,43 \%$ \\
\hline $\mathrm{L} 2$ & Learning & Informal & Post a discussion topic & $20,00 \%$ & $7,14 \%$ & $2,86 \%$ \\
\hline L3 & Learning & Informal & Meet a milestone & $30,00 \%$ & $10,71 \%$ & $4,29 \%$ \\
\hline L4 & Learning & Informal & Upload a learning object & $30,00 \%$ & $10,71 \%$ & $4,29 \%$ \\
\hline L5 & Learning & Informal & Enrich own learning object & $40,00 \%$ & $14,29 \%$ & $5,71 \%$ \\
\hline Lㄴ & Learning & Informal & Activity rate of recent contri & $10,00 \%$ & $3,57 \%$ & $1,43 \%$ \\
\hline L7 & Learning & Formal & EVAL: Self examination & $\overline{20}, \overline{0} \overline{\%}$ & $\overline{12,50 \%}$ & $\overline{5}, \overline{00 \%}$ \\
\hline L8 & Learning & Formal & EVAL: Continuous evalu & $40,00 \%$ & $25,00 \%$ & $10,00 \%$ \\
\hline$\underline{L 9}$ & Learning & Formal & EVAL: Self test & $20,00 \%$ & $12,50 \%$ & $5,00 \%$ \\
\hline \multirow[t]{7}{*}{ ID } & Category & Setting & Input & Abs & Rel 100 & Rel 40 \\
\hline & Interaction & & Input Interaction Subtotal & $100,00 \%$ & $100,00 \%$ & $40,00 \%$ \\
\hline & & & Input Interaction Informal & $50,00 \%$ & $50,00 \%$ & $20,00 \%$ \\
\hline & & & Input Interaction Formal & $50,00 \%$ & $50,00 \%$ & $20,00 \%$ \\
\hline & & & Subtotal Interaction & $230,00 \%$ & $100,00 \%$ & $40,00 \%$ \\
\hline & & & Subtotal Interaction Informal & $205,00 \%$ & $50,00 \%$ & $20,00 \%$ \\
\hline & & & Subtotal Interaction Formal & $25,00 \%$ & $50,00 \%$ & $20,00 \%$ \\
\hline$\overline{11}$ & Interaction & Informal & Complete the profile & $35,00 \%$ & $8,54 \%$ & $3,41 \%$ \\
\hline 12 & Interaction & Informal & Select top & $25,00 \%$ & $6,10 \%$ & $2,44 \%$ \\
\hline 13 & Interaction & Informal & Reply a & $20,00 \%$ & $4,88 \%$ & $1,95 \%$ \\
\hline 14 & Interaction & Informal & Rate & $15,00 \%$ & $3,66 \%$ & $1,46 \%$ \\
\hline 15 & Interaction & Informal & Reply a disci & $30,00 \%$ & $7,32 \%$ & $2,93 \%$ \\
\hline 16 & Interaction & Informal & Rate a discu & $15,00 \%$ & $3,66 \%$ & $1,46 \%$ \\
\hline 17 & Interaction & Informal & Discussion activity & $35,00 \%$ & $8,54 \%$ & $3,41 \%$ \\
\hline 18 & Interaction & Informal. & Enrich other's learning & $30,00 \%$ & $7,32 \%$ & $2,93 \%$ \\
\hline 19 & Interaction & Formal & Link from-to an external subje & $5,00 \%$ & $\overline{10}, \overline{0} \overline{\%}$ & $\overline{4}, \overline{00 \%}$ \\
\hline 110 & Interaction & Formal & Collaborative work rate & $20,00 \%$ & $40,00 \%$ & $16,00 \%$ \\
\hline \multirow[t]{7}{*}{ ID } & Category & Setting & Input & Abs & Rel 100 & Rel 20 \\
\hline & Men & & Input Mentoring Subtotal & $100,00 \%$ & $100,00 \%$ & $20,00 \%$ \\
\hline & & & Input Mentoring Informal & $50,00 \%$ & $50,00 \%$ & $10,00 \%$ \\
\hline & & & Input Mentoring Formal & $50,00 \%$ & $50,00 \%$ & $10,00 \%$ \\
\hline & & & Subtotal Mentoring & $285,00 \%$ & $100,00 \%$ & $20,00 \%$ \\
\hline & & & Subtotal Mentoring Informal & $105,00 \%$ & $50,00 \%$ & $10,00 \%$ \\
\hline & & & Subtotal Mentoring Formal & $180,00 \%$ & $50,00 \%$ & $10,00 \%$ \\
\hline M1 & Mentoring & Informal & Trust rate from a peer & $25,00 \%$ & $11,90 \%$ & $2,38 \%$ \\
\hline M2 & Mentoring & Informal & Trust rate from an expert & $30,00 \%$ & $14,29 \%$ & $2,86 \%$ \\
\hline M3 & Mentoring & Informal & Reputation rate from a & $20,00 \%$ & $9,52 \%$ & $1,90 \%$ \\
\hline M4 & Mentoring & Informal & Reputation rate from an expert & $30,00 \%$ & $14,29 \%$ & $2,86 \%$ \\
\hline $\bar{M} \overline{5}$ & Mentoring & Formal & EVAL: Final assessment & $\overline{6} 0,00 \%$ & $\overline{16}, \overline{6} \%$ & $\overline{3}, \overline{3} 3 \%$ \\
\hline M6 & Ment & Form & EVAL: Tracking asse & $60,00 \%$ & $16,67 \%$ & $3,33 \%$ \\
\hline M7 & Men & Form & Trust rate from a teacher & $20,00 \%$ & $5,56 \%$ & $1,11 \%$ \\
\hline M8 & Men & Formal & Reput & $10,00 \%$ & $2,78 \%$ & $0,56 \%$ \\
\hline M9 & Mentoring & Formal & pic rate & $10,00 \%$ & $2,78 \%$ & $0,56 \%$ \\
\hline M10 & Mentoring & Formal & Significan & $10,00 \%$ & $2,78 \%$ & $0,56 \%$ \\
\hline M11 & Mentoring & Formal & Quality of reply & $10,00 \%$ & $2,78 \%$ & $0,56 \%$ \\
\hline
\end{tabular}

\begin{tabular}{|l|c|c|}
\hline \multicolumn{2}{|c|}{ Strategy } & Check \\
\hline Cat-Setting & Weight & \\
\hline Informal & $50,00 \%$ & Ok \\
\hline Formal & $50,00 \%$ & \\
\hline & & \\
\hline Learning & $40,00 \%$ & Ok \\
\hline Interaction & $40,00 \%$ & \\
\hline Mentoring & $20,00 \%$ & \\
\hline
\end{tabular}

Fig. 4. LIME model for the application scenario. Settings, Inputs and Strategy 
This set of inputs gathers most of the requirements of the LIME model, including Trust, Reputation, Assessment, Evaluation, formal activities, and informal actions. In doing so, we select a representative amount of inputs, across a variety of types, which feed (back and forth) the LIME model. We have assigned the EVAL inputs to Formal settings, since the EVAL inputs in Informal settings would have required a specific assessment model for informal learning, which is not a topic of this research, although and interesting one for the future.

The system retrieves input data and provides recommendation once a week. Since the selected subject lasts 4 weeks (from July $2^{\text {nd }}$, to July $29^{\text {th }}$ ), we have established four milestones in months 8, 15, 22 and 29. These milestones store the specific data for every input and user incrementally, so that we can analyze the evolution of any specific user, with and without recommendation. At the end of the period (M29), every user in Group A (experimental) has received a considerable amount of recommendations, which might or might not lead to a higher performance, and to and improvement throughout the activities and actions in the Learning Environment.

About the timeline, the recommendations are provided through the milestones M8, M15, M22 and M29. However, there is no rule defined to adapt these recommendations to the user progress. Therefore, they have to be taken in close relation to the timeline. For instance, in M8, the recommendation R9 about Evaluation (see Table 4) will be likely provided to everyone, since there is little time since the beginning to the course up to M8 to carry out the activities and actions related to the Evaluation. However, in M15, and in $\mathrm{M} 22$, since the course is running for a longer time, it is expected that R9 will be provided to less people, decreasingly, until the final recommendation in M29, which will show the actual performance on Evaluation of every learner. Therefore, the recommendation has to be put in context of the timeline and the user (i.e. learner, tutor, and teacher) has to achieve a contextualized, appropriate reading, in order to act accordingly. Other potential contexts might be: the user status, in relation to previous subjects; the user status, in relation to the group; the user status, in relation to other groups of the same graduation; the group status, in relation to other groups of the same graduation; the group status in relation to historic records; et cetera.

Once the experiment is finished, we analyze the overall data, in order to extract group information, behavior patterns, abnormal actions, and other relevant information which will allow for a refinement of the LIME model and, if possible, the i-LIME software development and implementation.

This stored information is processed by the recommendation rules in DROOLS (language for rules processing), which takes the raw figures, applies the LIME model, and provides a recommendation on the learning itinerary. For our research, we have implemented a rule-set, adapted to this specific learning scenario and context. This rule-set must be defined by the learning designer (e.g. teacher) and it applies the LIME model based on the collected figures, and the style that the designer wants to reach, in addition to group goals and individual thresholds. In our case, the pseudo-code that describes the rules is as follows (Table 4):

TABLE 4: DEFINITION OF RULES

\begin{tabular}{|c|c|c|}
\hline RuleID & Applied to & RulePseudoCode \\
\hline R1 & $\begin{array}{l}\text { Input (e.g. L1 or } \\
\text { M3) }\end{array}$ & $\begin{array}{l}\text { IF any input is lower than maximum AND } \\
\text { higher than or equal to } 1 / 2 \text { maximum } \\
\text { THEN positive feedback about this } \\
\text { specific input }\end{array}$ \\
\hline R2 & Input & $\begin{array}{l}\text { IF any input is lower than } 1 / 2 \text { maximum } \\
\text { THEN warning about this specific input }\end{array}$ \\
\hline R3 & $\begin{array}{l}\text { Subset (Informal- } \\
\text { Formal) }\end{array}$ & $\begin{array}{l}\text { IF any subset of inputs in a category is } \\
\text { between maximum AND } 3 / 4 \text { of maximum } \\
\text { THEN positive feedback about the subset }\end{array}$ \\
\hline R4 & $\begin{array}{l}\text { Subset (Informal- } \\
\text { Formal) }\end{array}$ & $\begin{array}{l}\text { IF any subset of inputs in a category is } \\
\text { between } 1 / 2 \text { maximum AND } 3 / 4 \text { of } \\
\text { maximum THEN warning about the } \\
\text { subset to the learner and to the tutor }\end{array}$ \\
\hline R5 & $\begin{array}{l}\text { Subset (Informal- } \\
\text { Formal) }\end{array}$ & $\begin{array}{l}\text { IF any subset of inputs in a category is } \\
\text { lower than } 1 / 2 \text { maximum THEN warning to } \\
\text { the learner and to the teacher, } \\
\text { recommendation of interaction with others } \\
\text { and the tutor and the teacher, locking of } \\
\text { further activities in this category until the } \\
\text { threshold ( } 1 / 2 \text { maximum) is reached }\end{array}$ \\
\hline R6 & $\begin{array}{l}\text { Category (Learning, } \\
\text { Interaction, } \\
\text { Mentoring) }\end{array}$ & $\begin{array}{l}\text { IF any category is between maximum } \\
\text { AND } 3 / 4 \text { maximum THEN positive } \\
\text { feedback and recommendation of } \\
\text { complementary tasks }\end{array}$ \\
\hline R7 & $\begin{array}{l}\text { Category (Learning, } \\
\text { Interaction, } \\
\text { Mentoring) }\end{array}$ & $\begin{array}{l}\text { IF any category is between } 1 / 2 \text { maximum } \\
\text { AND } 3 / 4 \text { maximum THEN warning about } \\
\text { the category to the learner, the tutor and } \\
\text { the teacher; request of support from other } \\
\text { learners }\end{array}$ \\
\hline $\mathrm{R} 8$ & $\begin{array}{l}\text { Category (Learning, } \\
\text { Interaction, } \\
\text { Mentoring) }\end{array}$ & $\begin{array}{l}\text { IF any category is lower than } 1 / 2 \text { maximum } \\
\text { THEN warning to the learner and to the } \\
\text { teacher, recommendation of interaction } \\
\text { with others and the tutor and the teacher, } \\
\text { request of support from other learners, } \\
\text { locking of further activities in this } \\
\text { category until the threshold ( } 1 / 2 \\
\text { maximum) is reached }\end{array}$ \\
\hline R9 & EVAL & $\begin{array}{l}\text { IF any EVAL input is lower than } 1 / 2 \\
\text { maximum THEN locking of activity, } \\
\text { request of interactive session with teacher, } \\
\text { request of resubmission of activity-action }\end{array}$ \\
\hline
\end{tabular}

The specific coding of every rule looks like the following one, described for R1 (Table 5):

TABLE 5: EXAMPLED-PSEUDO CODE FOR RULE R1

\begin{tabular}{|l|l|l|}
\hline RuleID & Rule Coding & $\begin{array}{l}\text { Recommendation provided, } \\
\text { adapted to L1 }\end{array}$ \\
\hline R1 & $\begin{array}{l}\text { IF (L* OR I* OR M* < REL } \\
\text { X) AND (L* OR I* OR M* } \\
>=50 \% * \text { REL X) THEN } \\
\text { R1("Positive feedback to } \\
\text { USER") }\end{array}$ & $\begin{array}{l}\text { message" } \\
\end{array}$ \\
\hline
\end{tabular}

Technically, the raw data were stored in text files, which were translated in tables (XLS type) for easier representation, calculation, and analysis. A software application was created to analyze these files and extract the information from the XLS files. The particular scenario described here should be taken into account in order to interpret the obtained data. Each time the user executed an action foreseen in the LIME model, all the related information from that specific user was written into 
a log file. Every record is uniquely identified, and consists of basic ID information (i.e. date and timestamp of the event, action taken, user) and specific values provided according to the input (i.e. reputation, trust, assessment, other rates). There is an additional field with warnings, errors, and comments from the system. The information extracted from the log files was inserted into a database (XLS type) in order to organize the information and to make the information process easier. See Figure 5 for a simplified representation of the described application scenario in combination with the Adaptation Management System, which depicts the information flow from-to the end user.

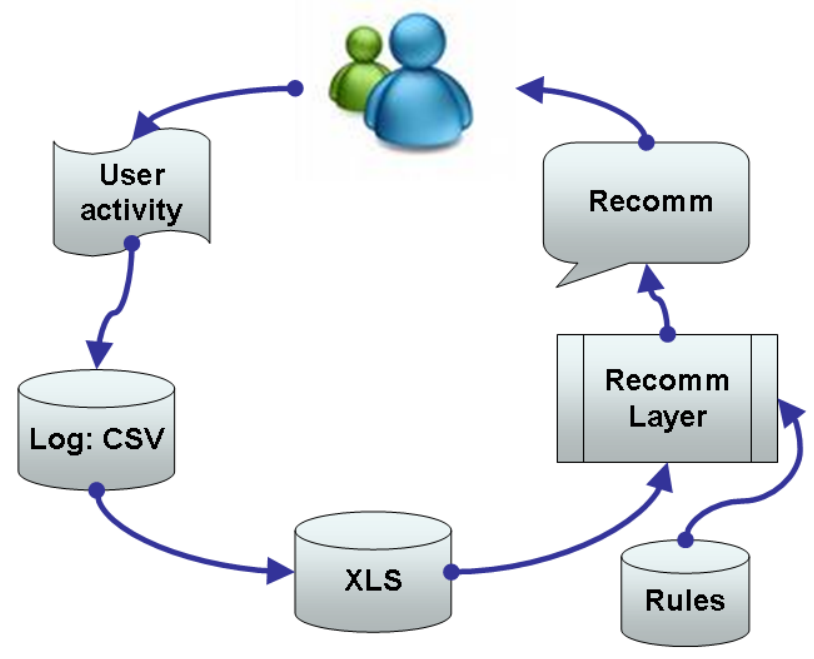

Fig. 5. Application scenario in combination with the AMS. Information flow

VII. RESULTS AND ANALYSIS OF THE APPLICATION SCENARIO

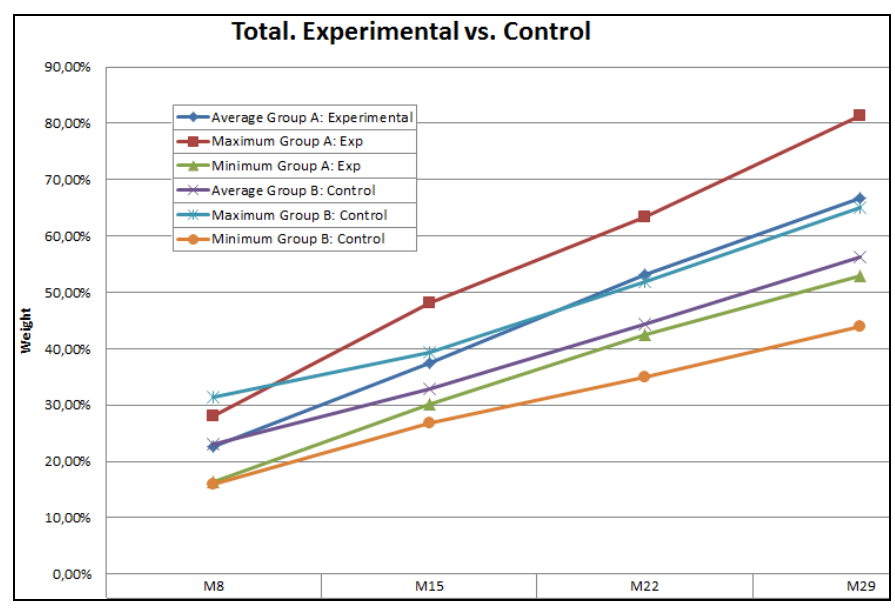

\begin{tabular}{|c|c|c|c|}
\hline M8 & M15 & M22 & M29 \\
\hline $22,61 \%$ & $37,47 \%$ & $53,09 \%$ & $66,72 \%$ \\
\hline $28,15 \%$ & $48,07 \%$ & $63,36 \%$ & $81,41 \%$ \\
\hline $16,42 \%$ & $30,12 \%$ & $42,48 \%$ & $52,88 \%$ \\
\hline $23,01 \%$ & $32,83 \%$ & $44,39 \%$ & $56,19 \%$ \\
\hline $31,32 \%$ & $39,28 \%$ & $51,94 \%$ & $65,06 \%$ \\
\hline $16,05 \%$ & $26,88 \%$ & $35,06 \%$ & $44,04 \%$ \\
\hline
\end{tabular}

Fig. 6. Total results. Experimental group versus Control group

In Figure 6, we compare final, general results of the experimental group (A) in opposition to the control group (B). We provide data for the four milestones (M8, M15, M22, M29), and three variables per each: Maximum score,
Minimum Score, and Average Score. Therefore, we analyze a six-line web along four weeks. As expected from interviews with end users and the Delphi study, the final score is higher with the experimental group (A:66,72\% vs. B:56,19\%, in M29, over a $100 \%$ top). However, the crossed lines show a higher average position of the control group in M8 (A:22,61\% vs. B:23,01\%), before a linear increase up to M29. In addition, the maximum score in M8 is higher at the control group (A:28,15\%; B:31,32\%). These two higher scores at the control group at the beginning show a symptomatic progress of the impact from the recommendation system: although in the beginning $\mathrm{A}$ and $\mathrm{B}$ can be alike, or even $\mathrm{B}$ shows a higher rank, the systematic application of recommendations through the i-LIME environment overcomes the evolution without the LIME model

\section{VIII.CONCLUSIONS AND FUTURE WORK}

L.I.M.E. provides an optimized formula which allows for finding the balance between all the inputs related to the online learning, as in our vision. The model describes the right weight for every input, directly related to the effect to achieve along the process and every role. Based on the ground objectives, the learning scenario will define the required interaction between inputs, roles, categories and settings. The model is based on behavior, performance and the relation among the end user, himself and the peers. Furthermore, there are four main pillars or vectors: Learning, Interaction, Mentoring and Evaluation (aka L.I.M.E.). Each of them provides a key to define the relation of the user within the mode, which is translated into a set of interconnected rules. Based on what the user does in the system, and how this web is weaved, the model provides the user with personalised guidance, dynamic along the timeline, which allows for a stable tutoring support along the learning process.

In order to validate the L.I.M.E. model, we have designed and implemented a learning scenario, during 4 weeks, and counting 2 groups (experimental-A and control-B) of 24 members each. The application of the model to the described scenario shows a clear and positive progress of the users in group A, those who received recommendations by the system. The overall average of inputs, categories and students shows a final positive difference of $10,53 \%$ between the experimental group and the control group $(66,72 \%-56,19 \%)$, in addition to a maximum difference between corners of $37,37 \%(81,41 \%$ $44,04 \%)$. These results become a tangible proof for the success of the L.I.M.E. model, based on a large number of objective measurements. They back up the conceptual design from a practical experience. Furthermore, they support the combination of inputs and categories provided by L.I.M.E., which facilitates personalized counseling to the end-user, leading to an improvement of his average performance, implemented in the context of a thematic, restricted social network, and learning scenario which engages formal and informal settings, through learning activities and user interaction.

Future work points out at an early definition phase that 
should take into account every single role (i.e. student, teacher, admin, et cetera). This involvement should not come from the instructional designer only, but from actual users from every target group. In doing so, the designer builds an ecosystem which plays with every actor from inside, and not only a scenario in which the users are included from outside. In addition, the model would benefit of a more precise balance between settings, inputs and categories. The combination of these is crucial for a good use of the system. In our application case, we use a neutral approach, so that we did not influence the results because of an early selection of these elements. However, no matter what the selection is, since it always affects the result, even for being neutral. A clear definition of the implications and co-lateral effects of each configuration would better support the match between objectives and expectations from students, tutors, and instructional designers

\section{ACKNOLEDGMENT}

This paper is being supported by the EduMotion Project (grant agreement 315568), funded by the Seventh Framework Programme of the European Commission, under the Research for SMEs line (R4SME); the TELMA project (TSI-0201102009-85) of the Spanish Ministry of Industry, Tourism and Trade; and UNIR Research (http://research.unir.net), private research initiative which supports the Research Group on eLearning and Social Networks TELSOCK at the university. Finally, we thank Prof. Dr. José Luis Rodríguez Illera and Dr. Mario Barajas, from University of Barcelona, for their support.

\section{REFERENCES}

[1] R. A. Bjork, "Assessing our own competence: Heuristics and illusions," in Attention and performance XVII. Cognitive regulation of performance: Interaction of theory and application, D. Gopher and A. Koriat, Eds. Cambridge, MA: MIT Press, 1999, pp. 435-459.

[2] V. Romero and D. Burgos, "Meta-Mender: A meta-rule based recommendation system for educational applications," presented at Proceedings of the Workshop on Recommender Systems for Technology Enhanced Learning, RecsysTEL-2010, Barcelona, Spain, 2010.

[3] V. Romero, D. Burgos, and A. Pardo, "Meta-rule based Recommender Systems for Educational Applications," in Educational Recommender Systems and Technologies: Practices and Challenges, O. Santos and J. Boticario, Eds.: Information Science-Idea Group, 2011.

[4] B. White and J. Frederiksen, "A theoretical framework and approach for fostering metacognitive development," Educational Psychologist, vol. 40, pp. 211-223, 2005.

[5] G. Linden, B. Smith, and Y. J., "Amazon.com recommendations: Itemto-item collaborative filtering," Internet Computing IEEE, vol. 7, pp. 76-80, 2003.

[6] B. Marlin, "Modeling user rating profiles for collaborative filtering," in Advances in neural information processing systems, S. Thrun, L. K. Saul, and B. Schölkopf, Eds. Cambridge, MA: MIT Press, 2003, pp. 627-634.

[7] S. Y. Chen and G. D. Magoulas, Adaptable and Adaptive Hypermedia Systems. Hershey, PA: IRM Press, 2005.

[8] K. I. Ghauth and N. A. Abdullah, "Learning materials recommendation using good learners' ratings and content-based filtering," Education Technology Research and Development, In press.

[9] T. Kerkiri, A. Manitsaris, and A. Mavridou, Reputation metadata for recommending personalized e-learning resources. Uxbridge: IEEE Computer Society, 2007.
[10] C. Romero, S. Ventura, P. D. De Bra, and C. D. Castro, "Discovering prediction rules in AHA! courses.," presented at 9th International User Modeling Conference, 2003.

[11] D. Burgos, C. Tattersall, and R. Koper, "How to represent adaptation in eLearning with IMS Learning Design," Interactive Learning Environments, vol. 15, pp. 161-170, 2007.

[12] J. J. Rocchio, Relevance feedback in information retrieval, in the SMART Retrieval System. Experiments in Automatic Document Processing. Englewood Cliffs, NJ: Prentice Hall, Inc., 1971.

[13] P. Sánchez-Gonzáleza, I. Oropesaa, V. Romeroc, A. Fernándeza, A. Albaceted, E. Asenjoe, J. Nogueraf, F. Sánchez-Margallog, D. Burgosc, and E. J. Gómez, "TELMA: technology enhanced learning environment for Minimally Invasive Surgery," Procedia Computer Science, vol. 00, 2010.

[14] Romero L, Gutiérrez M, Caliusco ML. Conceptualizing the e-Learning Assessment Domain using an Ontology Network. International Journal of Interactive Multimedia and Artificial Intelligence. 2012;1 (Special Issue on Intelligent Systems and Applications):20-8

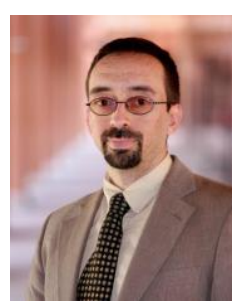

Prof. Dr. Daniel Burgos works as Vice-chancellor for Research \& Technology, and Director of Engineering at the International University of La Rioja (www.unir.net, http://research.unir.net). He also holds the Technology Innovation Chair at the same university. Previously he worked as Director of Education Sector and Head of User Experience Lab in the Research \& Innovation Department of Atos, Spain, since 2007; and as an Assistant Professor at Open Universiteit Nederland before that.

His research interests are mainly focused on Adaptive and Informal eLearning, Learning \& Social Networks, eGames, and eLearning Specifications. He is or has been involved in a number of European funded R\&D projects like, i.e. Intuitel, Hotel, Edumotion, Inspiring Science Education Stellar, Gala, IntelLEO, Go-MyLife, Grapple, Unfold, ProLearn, TenCompetence, EU4ALL, NiHao, Kaleidoscope, Sister, ComeIn, et cetera. Prof. Dr. Burgos holds degrees in Communication (PhD), Computer Science (Dr. Ing), Education, and Business Administration. Furthermore, he is member of a number of Executive Boards of associations and professional clusters focused on educational technology and eLearning innovation like, i.e. Telearc, Telspain, Menon Network, Adie, and others. 